\title{
Effect of contralateral cold on pain tolerance'
}

BERNARD BLITZ and ALBERT J. DINNERSTEIN, New York Medical College, New York, N. Y. 10029

Fourteen Ss reported pain threshold and quit point in trials in which either right, left, or both hands were immersed in an ice water mixture. The contralateral cold stimulation resulted in significant elevation in both pain threshold and quit point for both right and left hands. The data also indicated that even nonpainful contralateral cold elevated pain threshold; this latter effect showed adaptation.

An earlier study has shown contralateral cold stimulation to have the effect of elevating thresholds for pain and discomfort (Blitz \& Dinnerstein, 1968). This finding confirmed other observations of the pain attenuating effects of cold applied to sites distant from that of the noxious stimulation (Gammon \& Starr, 1941; Parsons \& Goetzl, 1945). The present study is a further exploration of this phenomenon. It provides information regarding the pain attenuating effects of contralateral cold stimulation (1) for noxious stimulation at pain tolerance levels (quit point), (2) for the condition where the pain stimulus is applied to the right or left hand, and (3) when the contralateral cold is at a nonpainful level.

\section{METHOD}

The Ss were 14 paid male volunteers from a college population. Each $S$ was given a series of 12 trials in each of which he placed the fingers of one or both of his hands (up to the palm joint) in a tank containing an ice water mixture gently circulating, at close to $0 \mathrm{deg} C$. For each trial the $\mathrm{S}$ reported pain threshold (for trials involving both hands, he reported pain threshold for each hand separately) and then kept his hand (or hands) in the water as long as possible (quit point). If on any trial the $S$ reported his hand feeling numb before reaching quit point, he was told to remove that hand from the ice water and his time at that point was treated as quit point.

The sequence of the trial conditions for half the Ss, assigned at random, was as follows: (1) left hand, (2) right hand, (3) both hands, (4) both hands, (5) right hand, and (6) left hand. This sequence was repeated in another block of six trials. The other half of the Ss had an identical sequence except the order of left and right hand trials was reversed.

Between each trial S placed both hands in a tank of warm water at $35 \mathrm{deg} C$ for $2 \mathrm{~min}$, except for the period prior to the initial trial, for which the time was $1 \mathrm{~min}$. For the Both Hands trial, when $S$ reached the quit point for one hand, he took it out of the ice water and kept it in the air until he reached quit point for the other hand. Room temperature was maintained at $25 \mathrm{deg} C$.

\section{RESULTS}

The mean pain threshold and quit point (in seconds) for each hand for the single hand and both hand trials are shown in Fig. 1.

The effect of contralateral cold was tested as follows. For each block of six trials a "baseline score" was computed for each S for each hand by averaging his pain threshold (or quit point) scores for the One Hand trials preceding and following (i.e., Trials 1 and 6 , or 2 and 5) the Both Hands trials (Trials 3 and 4 ). The "baseline score" was then subtracted from the mean pain threshold (or quit point) for that hand for the two Both Hands trials, yielding a difference score. This difference score was divided by the baseline scores to yield a measure of "per cent increment." This measure was obtained for each hand, for pain threshold and quit point, for each of the two blocks of trials. The differences in these per cent increment scores between Blocks 1 and 2 were not significant (Wilcoxon Signed Ranks Test). Scores for Blocks 1 and 2 were therefore combined by averaging.

The mean and median per cent increment scores for pain threshold were as follows: right hand, mean $=+.51$, median $=+.21$ $(p<.01)$; left hand, mean $=+.61$, median $=+.39(p<.01)$. The $p$ values were derived by use of the Wilcoxon Signed Ranks Test.

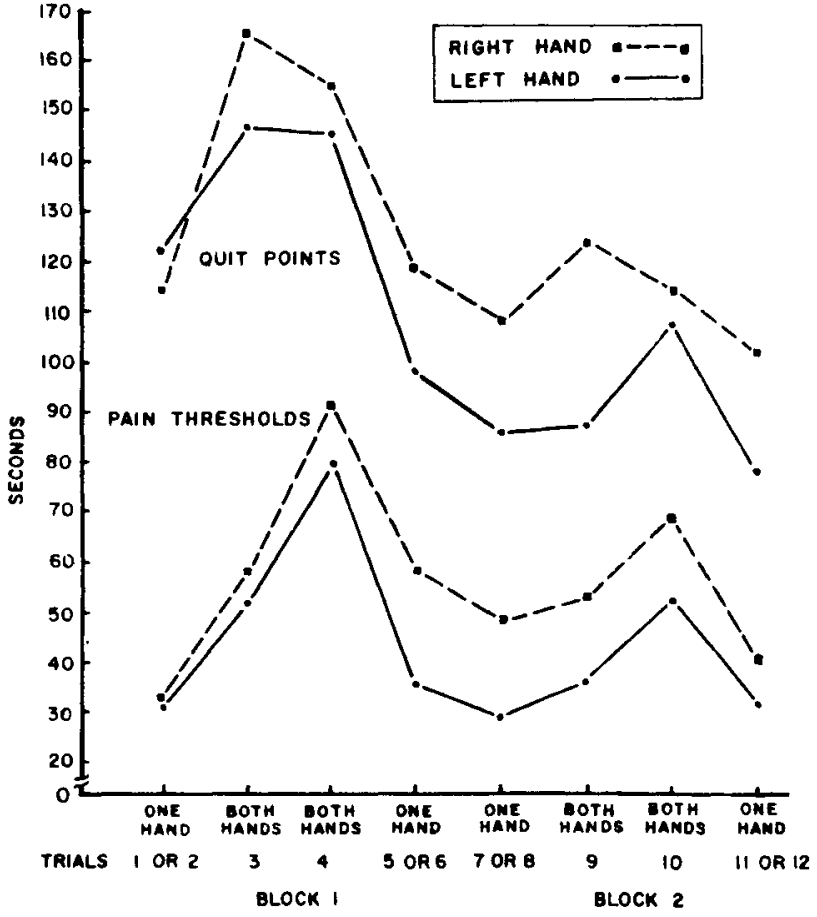

Fig. 1. Mean pain threshold and quit point for right and left hands for each One Hand and Both Hands trial. (Note: The One Hand trial scores are based on the combination of Ss starting with trial sequence LR. . and those with sequence RL... For example, the mean pain threshold for the right hand for the first One Hand trial is based on scores for seven Ss for Trial 1 and seven Ss for Trial 2.)

For quit point the mean and median per cent increment scores were as follows: right hand, mean $=+.21$, median $=+.15(p<.05)$; left hand, mean $=+.20$, median $=+.11(p<.05)$. The differences between the right and left hand per cent increment scores for pain threshold and for quit point were not significant.

The effect of contralateral cold on pain threshold as measured above does not separate the effect of contralateral cold, per se, from that of contralateral pain; for in the Both Hands trial once one hand has reached pain threshold the threshold for the other hand may be a consequence of the contralateral cold and/or of the pain. Therefore, to isolate the effect of contralateral cold from that of pain, the following procedure was used. For each of the Both Hands trials only the pain threshold for the hand with the lower threshold was considered. Thus, since the opposite hand, that with the higher threshold, had not reached the level of "pain," the pain threshold of the hand with the lower threshold reflected only the effect of contralateral cold. For each of the Both Hands trials the increment above baseline for the hand with the lower threshold was converted to per cent increment and these scores were averaged for each S. A number of Ss reported pain simultaneously for both hands (seven $\delta s$ in Block 1 and two Ss in Block 2). In this case the per cent increments for both hands were used in this analysis, for these scores were not a function of prior contralateral pain. (It should be noted that, due to random errors of sampling, this procedure would statistically underestimate the effect of contralateral cold.)

Despite the above bias, the per cent increment for the "lower threshold" hand for pain threshold for Block 1 was: mean $=+.60$, median $=.13(p<.01)$ and for Block 2 , mean $=+.04$, median $=-.06$ (not significant). The difference between Blocks 1 and 2 was significant $(\mathrm{p}<.05)$. 


\section{DISCUSSION}

These results confirm and extend the findings of the authors' earlier study (Blitz \& Dinnerstein. 1968). Not only does contralateral cold elevate pain threshold for cold stimulation. it also elevates quit point. These effects are obtained with either the right or the left hand serving as the "masking hand": however. the present sample was too small to look for the differential effects of dominance.

The present study also provided some data indicating that contralateral cold, per se, in the absence of pain will elevate pain threshold. This effect was found only in the earlier block of trials implying possible adaptation. The fact that there was no significant difference between Blocks 1 and 2 for per cent increment in pain threshold when all trials are considered. suggests that adaptation is less likely when contralateral cold produced pain.

There are a number of alternative explanations for the present results. These are: (1) spinal inhibitory processes (Melzack \& Wall. 1965 , (2) perceptual processes, akin to distraction. which weaken the "urgency" or salience of pain (Duncker. 1937), and (3) peripheral vascular effects induced by contralateral cold stimulation which interfere with the local mechanisms responsible for pain. The possible role of vascular effects is suggested by a study by Wagoner \& Havemann (1936) which found contralateral cold (23 deg C) to elevate threshold for cold; this finding is explained by vasoconstriction induced by contralateral cold. However. the role of such a mechanism in the present findings is not clear.

\section{REFERENCES}

BLITZ. B.. \& DINNERSTEIN, A. J. Pain attenuation by contralateral cold stimulation. Psychonomic Science, 1968. 10. 395-396.

DUNCKER. K. Some preliminary experiments on the mutual interference of pains. Psychologische Forschung, 1937. 21, 311-326.

GAMMON, G. D.. \& STARR, I. Studies on the relief of pain by counterirritation. Journal of Clinical Investigation. 1941, 20,13-20.

MELZACK. R.. \& WALL. P. D. Pain mechanisms: A new theory. Science. 1965. 150.971.979.

PARSONS. C. M.. \& GOETZL, F. R. Effect on induced pain on pain threshold. Proceedings of the Society of Experimental Biology and Medicine, 1945. 60.327-329.

WAGONER. K. S., \& HAVEMANN. E. The effect of thermal stimulation of one hand upon the temperature linen in the contralateral hand. Journal of Psychology, 1963, 2, 441 449.

\section{NOTE}

1. This research was supported by Grant No. MH 12097 from the National Institute of Mental Health and Grant No. RD 1372.P from the Vocational Rehabilitation Administration. The authors are indebted to Dr. M. Lowenthal, principal investigator of the latter grant and also to Peter Klein and Eileen Brady for their assistance in collecting and analyzing the data. 